\title{
PPE shortages leaving staff at risk and urgent care system in jeopardy
}

A poll by the BDA of dentists staffing the emerging network of Urgent Dental Care centres suggests a third of sites in England remain inactive, with more than half reporting that their operational status is being impacted by a lack of Personal Protective Equipment (PPE).

Following the suspension of routine care the network was meant to provide urgent care for dental patients during the COVID-19 pandemic.

The BDA's poll has found:

$\rightarrow$ Over half (54\%) of dentists responding in England report PPE shortages are impacting on the operational status of sites. Only 1 in 5 dentists in Scotland are reporting the same issues $\rightarrow$ Nearly two-thirds of dentists at sites in England report shortages of FFP3 masks and gowns

$\rightarrow 32 \%$ of dentists at sites in England designated to use FFP3 masks for Aerosol Generating Procedures have yet to be fit-tested. 52\% report shortages of the vital testing spray required to ensure the safe operation of these masks

$\rightarrow$ Some $62 \%$ of respondents in England report their sites as active. This rises to over $98 \%$ in devolved nations

$\rightarrow 58 \%$ of dentists report they do not feel fully protected at the sites they are working at. Official guidance continues to state even COVID-19 positive should be treated using basic PPE.

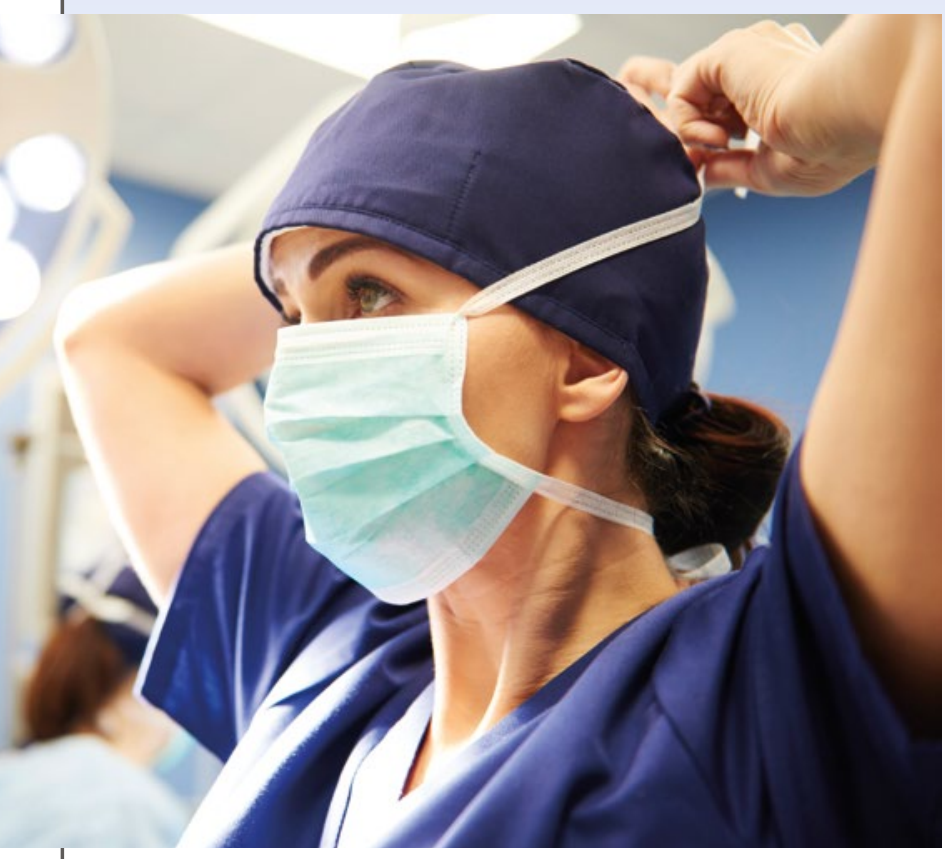

The slow roll-out in England has seen millions left without access to urgent care across whole regions. Yorkshire and Humber, one of the last regions to start getting a service up and running in recent days, are now reportedly already struggling to manage demand for urgent care, which includes cases of broken teeth, and abscesses, which can be life-threatening if left untreated.

Despite progress on bringing sites live, chronic shortages of PPE, together with a lack of training and fit testing, continues to leave many teams unable to treat patients.

Sites in the East Midlands have reported kit being requisitioned for use elsewhere in the health service. The BDA understands the scale of these shortages has led some dentists in the South East to actively explore alternative sources of PPE, including non-medical equipment, such as scuba masks.

NHS England had previously operated a distinction between 'hot' sites for COVID-19 symptomatic patients and 'cold' sites for treatment of other patients, including vulnerable populations. While that approach has now been dropped, it has left limited supplies of key PPE concentrated in a relatively small number of hot sites.

Active centres also appear to be operating at significantly lower than expected capacity, given the need to follow stringent decontamination procedures and social distancing guidelines, with reported patient volumes falling from forty patients per surgery per day pre-pandemic to eight. Where Aerosol Generating Procedures are used there is a need to leave surgeries fallow for an hour post-treatment, to minimise the risk of cross-infection.

Dentist leaders have accused NHS England of basing PPE guidance for staff not on risk, but on the limitations placed by the current shortage across the NHS, and urged a national effort to up production and distribution for all NHS front line staff.

\section{Pay dispute resolution service at work on first batch of cases}

The BDA has begun of the process of resolving the first pay disputes between and associates over pay during the COVID-19 pandemic.

Following successful lobbying corporate bodies and practice owners with NHS contracts will continue to be paid assuming certain conditions are met. One of those is that they are obliged to pass on income due to associates. Practices are also required to support the wider NHS pandemic effort, and this is likely to involve associates if NHS money is to be passed on.

The service's primary aim is to ensure that any issues are resolved in a fair and satisfactory way for everyone involved and so that effective working relationships are maintained.

Chair of the BDA's Associate Group Sarah Canavan said: 'The overwhelming majority of corporates and practice owners are acting in line with the deals we've struck with governments. But in those rare cases where this isn't happening we are already working to resolve matters swiftly and constructively.

'We are setting out to help all parties reach a fair agreement in line with NHS rules. At this difficult time, we are offering associates potentially the only route they have for real clarity on their pay'

New cases can be logged at https:// www.bda.org/advice/Coronavirus/Pages/ associates-coronavirus.aspx. 